\title{
3D Computational Mechanical Analysis for Human Atherosclerotic Plaques Using MRI-Based Models with Fluid-Structure Interactions
}

\author{
Dalin Tang, ${ }^{1}$ Chun Yang, ${ }^{2}$ Jie Zheng, ${ }^{3}$ Pamela K. Woodard, ${ }^{3}$ Gregorio A. Sicard, ${ }^{4}$ \\ Jeffrey E. Saffitz, ${ }^{5}$ Shunichi Kobayashi, ${ }^{6}$ Thomas K Pilgram, ${ }^{3}$ and Chun Yuan ${ }^{7}$ \\ ${ }^{1}$ Mathematical Sciences Department, Worcester Polytechnic Institute, \\ Worcester, MA 01609, USA, dtang@wpi . edu \\ ${ }^{2}$ Mathematics Dept, Beijing Normal University, Beijing, China, \\ chyang0@btamail.net.cn \\ ${ }^{3}$ Mallinkcrodt Institute of Radiology, Washington University, St. Louis, MO 63110, USA \\ Jie Zheng: zhengj @mir . wust 1 . edu; Pamela Woodard: \\ woodardp@mir.wustl.edu \\ ${ }^{4}$ Department of Surgery, Washington University, St. Louis, MO 63110, USA, \\ sicardg@msnotes.wustl.edu \\ ${ }^{5}$ Dept of Pathology, Washington Universty, St. Louis, MO 63110, USA, \\ saffitzapathbox.wustl.edu \\ ${ }^{6}$ Dept. of Functional Machinery and Mechanics, Shinshu Univ., Nagano, Japan, \\ shukoba@giptc.shinshu-u.ac.jp \\ ${ }^{7}$ Deparment of Radiology, University of Washington, Seattle, WA 98195, USA, \\ cyuan@u.washington.edu
}

\begin{abstract}
Atherosclerotic plaques may rupture without warning and cause acute cardiovascular syndromes such as heart attack and stroke. It is believed that mechanical forces play an important role in plaque progression and rupture. A three-dimensional (3D) MRI-based finite-element model with multicomponent plaque structure and fluid-structure interactions (FSI) is introduced to perform mechanical analysis for human atherosclerotic plaques and identify critical flow and stress/strain conditions which may be related to plaque rupture. The coupled fluid and structure models are solved by ADINA, a well-tested finite-element package. Our results indicate that pressure conditions, plaque structure, component size and location, material properties, and model assumptions all have considerable effects on flow and plaque stress/strain behaviors. Large-scale patient studies are needed to validate the computational findings. This FSI model provides more complete stress/strain analysis and better interpretation of information from MR images and may lead to more accurate plaque vulnerability assessment and rupture predictions.
\end{abstract}

\section{Introduction}

Cardiovascular disease (CVD) is the No. 1 killer in the developed countries and is responsible for millions of deaths and disabilities every year. Atherosclerotic plaques may rupture without warning and cause subsequential acute syndromes such as 
myocardial infarction and cerebral stroke. A large number of victims of the disease who are apparently healthy die suddenly without prior symptoms. Available screening and diagnostic methods are insufficient to identify the victims before the event occurs [4]. Accurate methods are needed to identify vulnerable plaques that are prone to rupture and quantify conditions under which plaque rupture may occur.

MRI technologies have been developed to quantify non-invasively plaque size, shape, and plaque components (fibrous, lipid, and calcification/inflammation) [9]. In addition to plaque morphology and components, mechanical forces clearly play an important role in the rupture process. Mechanical analysis based on MR images has been proposed but is mainly limited to structure-only (2D or 3D) or flow-only (3D) models due to complexity of the problem [3,8]. Accurate flow and stress/strain analysis for plaque rupture predictions using 3D computational modeling with fluidstructure interactions (FSI) based on 3D MRI human plaque morphology and constituents is lacking in the literature.

In this paper, a finite-element 3D FSI model is introduced based on realistic human atherosclerotic plaque morphology and components to perform flow and plaque stress/strain analysis and quantify critical stress/strain conditions under which plaque rupture is likely to occur. 3D plaque geometry will be re-constructed from MR images. Vessel and plaque material properties from our own in vitro measurements [6] and existing literature [3,8] are used in the solid models. A typical pressure profile for human internal carotid artery was used to observe stress/strain behavior under pulsating pressure. The coupled fluid and solid models are solved by a finite element package ADINA which is capable of handling multi-physics models with fluid-structure interactions [1,2]. Sensitivity analysis was performed to quantify the effects of controlling factors (pressure condition, material properties, plaque structure, lipid size, plaque cap thickness, calcification, axial stretch) on critical plaque stress/strain conditions (maxima and minima of stress/strain values and their variations corresponding to various changes). Critical stress/strain values were compared with semi-quantitative histopathological plaque vulnerability assessment and some indicative correlation patterns were found.

\section{The Computational Model and Method}

\subsection{D Re-construction of Plaque Geometry Based on MRI Data}

3D ex vivo MRI data sets obtained from human atherosclerotic plaques consisting of 32-64 2D slices with high resolution $(0.1 \mathrm{~mm} \times 0.1 \mathrm{~mm} \times 0.5 \mathrm{~mm})$ were read by VTK [5] and 3D plaque geometry and mesh were re-constructed following the procedure described in [7]. Intensive interactions and additional programming from the operator/researcher were needed due to the complexity of plaque morphology and components. Boundary lines for various plaque components were generated according to segmentation data ranges validated by histological analysis. Fig. 1 shows 24 MRI slices (selected from a set of 36 slices) of a cadaveric plaque sample, plaque component contour plots based on histological segmentation data, and the reconstructed $3 \mathrm{D}$ geometry. The diameter of the vessel is about 5-6 mm. Resolution is 
$0.25 \mathrm{~mm} \times 0.23 \mathrm{~mm} \times 0.5 \mathrm{~mm}$. Some smoothing was applied. The vessel was extended uniformly at both ends by $3 \mathrm{~cm}$ and $6 \mathrm{~cm}$ respectively so that it became long enough for our simulations.

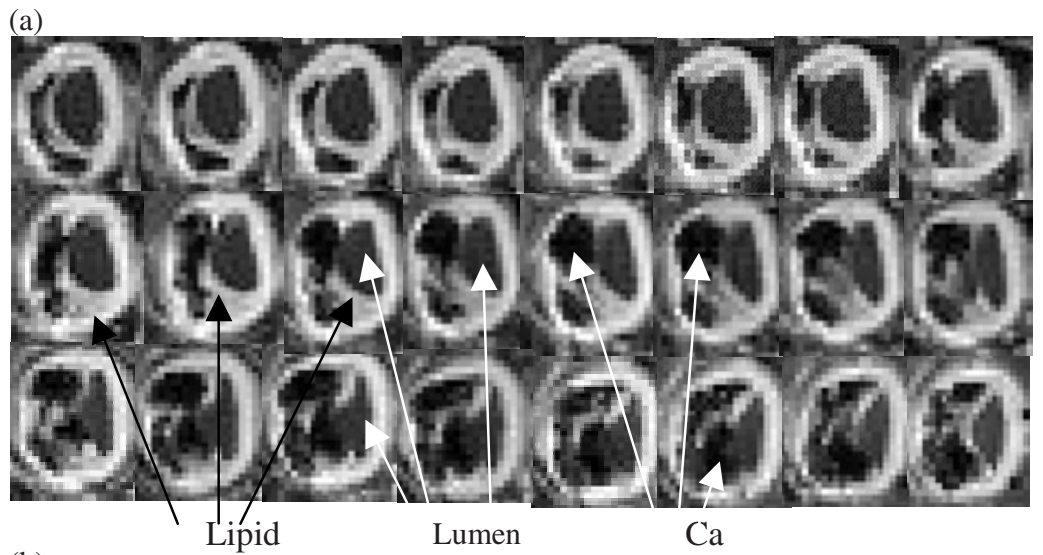

(b)

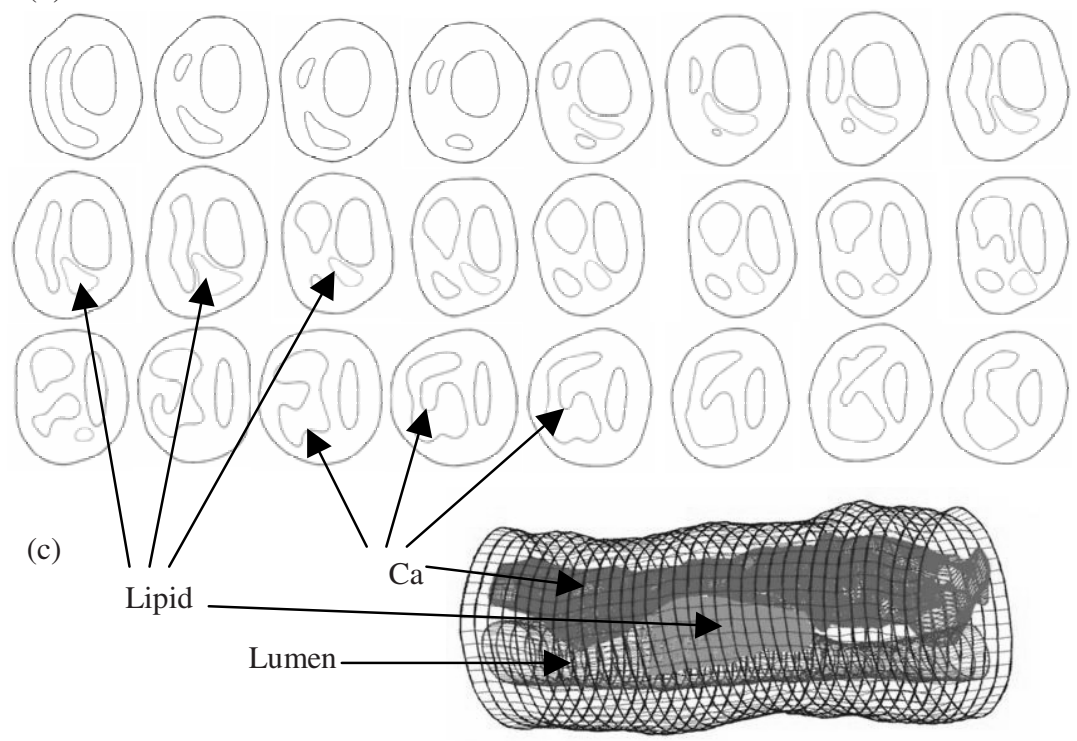

Fig. 1. A cadaveric plaque sample with large calcification block and a lipid pool. a) Selected MR images from a 36-slice set (S9-S33, from left to right); b) Component segmentations of MR images based on histopathological data. Some smoothing was applied; c) Re-constructed $3 \mathrm{D}$ plaque geometry. The position of the vessel is rotated for better viewing

\subsection{The Solid and Fluid Models}

Both artery wall and plaque components in the plaque were assumed to be hyperelastic, isotropic, incompressible and homogeneous. The flow was assumed to be laminar, Newtonian, viscous and incompressible. The incompressible Navier- 
Stokes equations with arbitrary Lagrangian-Eulerian (ALE) formulation were used as the governing equations which are suitable for FSI problems with frequent mesh adjustments. No-slip condition was assumed at all interfaces. Putting these together, we have (summation convention is used):

$$
\begin{aligned}
& \rho\left(\partial \mathbf{u} / \partial \mathrm{t}+\left(\left(\mathbf{u}-\mathbf{u}_{\mathrm{g}}\right) \cdot \nabla\right) \mathbf{u}\right)=-\nabla \mathrm{p}+\mu \nabla^{2} \mathbf{u}, \nabla \cdot \mathbf{u}=0, \\
& \left.\mathbf{u}\right|_{\Gamma}=\partial \mathbf{x} / \partial \mathrm{t}, \partial \mathbf{u} /\left.\partial \mathrm{n}\right|_{\text {inlet, outlet }}=0, \\
& \left.\mathrm{p}\right|_{\text {inlet }}=\mathrm{p}_{\mathrm{in}}(\mathrm{t}),\left.\quad \mathrm{p}\right|_{\text {outlet }}=\mathrm{p}_{\text {out }}(\mathrm{t}), \\
& \rho \mathrm{v}_{\mathrm{i}, \mathrm{tt}}=\sigma_{\mathrm{ij}, \mathrm{j}}, \mathrm{i}, \mathrm{j}=1,2,3 ; \text { sum over } \mathrm{j}, \quad \quad \text { (eq. of motion for solids) } \\
& \varepsilon_{\mathrm{ij}}=\left(\mathrm{v}_{\mathrm{i}, \mathrm{j}}+\mathrm{v}_{\mathrm{j}, \mathrm{i}}\right) / 2, \mathrm{i}, \mathrm{j}=1,2,3, \quad \text { (strain-displacement) } \\
& \left.\sigma_{\mathrm{ij}} \cdot \mathrm{n}_{\mathrm{j}}\right|_{\text {out_wall }}=0,\left.\quad \sigma_{\mathrm{ij}}^{\mathrm{r}} \cdot \mathrm{n}_{\mathrm{j}}\right|_{\text {interface }}=\left.\sigma_{\mathrm{ij}}^{\mathrm{s}} \cdot \mathrm{n}_{\mathrm{j}}\right|_{\text {interface, }}
\end{aligned}
$$

where $\mathbf{u}$ and $\mathrm{p}$ are fluid velocity and pressure, $\mathbf{u}_{\mathrm{g}}$ is mesh velocity, $\Gamma$ stands for vessel inner boundary, $f \cdot, j$ stands for derivative of $f$ with respect to the jth variable, $\boldsymbol{\sigma}$ is stress tensor (superscripts indicate different materials), $\varepsilon$ is strain tensor, $\mathbf{v}$ is solid displacement vector. The 3D nonlinear modified Mooney-Rivlin (M-R) model was used to describe the material properties of the vessel wall and plaque components $[1,2]$. The strain energy function is given by,

$$
\begin{aligned}
& \mathrm{W}=\mathrm{c}_{1}\left(\mathrm{I}_{1}-3\right)+\mathrm{c}_{2}\left(\mathrm{I}_{2}-3\right)+\mathrm{D}_{1}\left[\exp \left(\mathrm{D}_{2}\left(\mathrm{I}_{1}-3\right)\right)-1\right], \\
& \mathrm{I}_{1}=\sum \mathrm{C}_{\mathrm{ii}}, \mathrm{I}_{2}=1 / 2\left[\mathrm{I}_{1}^{2}-\mathrm{C}_{\mathrm{ij}} \mathrm{C}_{\mathrm{ij}}\right],
\end{aligned}
$$

where $I_{1}$ and $I_{2}$ are the first and second strain invariants [1,2], $c_{i}$ and $D_{i}$ are material constants. The stress/strain relations can be found by:

$$
\sigma_{\mathrm{ij}}=\left(\partial \mathrm{W} / \partial \varepsilon_{\mathrm{ij}}+\partial \mathrm{W} / \partial \varepsilon_{\mathrm{ji}}\right) / 2,
$$

where $\sigma_{\mathrm{ij}}$ are the second Piola-Kirchhoff stresses, $\varepsilon_{\mathrm{ji}}$ are the Green-Lagrange strains.

In this paper, the following values were chosen to match experimental data and existing literature [3,6,8]: Artery wall (including fibrous cap): $c_{1}=92,000 \mathrm{dyn} \bullet \mathrm{cm}^{-2}$, $\mathrm{c}_{2}=0, \mathrm{D}_{1}=36,000$ dyn $\mathrm{cm}^{-2}, \mathrm{D}_{2}=2$; Lipid: $\mathrm{c}_{1}=5,000 \mathrm{dyn} \bullet \mathrm{cm}^{-2}, \mathrm{c}_{2}=0, \mathrm{D}_{1}=5,000 \mathrm{dyn} \bullet \mathrm{cm}^{-2}$, $\mathrm{D}_{2}=1.5$; Calcification: $\mathrm{c}_{1}=920,000$ dyn $\mathrm{cm}^{-2}, \mathrm{c}_{2}=0, \mathrm{D}_{1}=360,000 \mathrm{dyn} \bullet \mathrm{cm}^{-2}, \mathrm{D}_{2}=2$.

\subsection{Solution Method}

The fully coupled fluid and structure models were solved by a commercial finiteelement package ADINA (ADINA R \& D, Inc., Watertown, MA, USA) which has been tested by hundreds of real-life applications [2] and has been used by Tang in the last several years [6,7]. ADINA uses unstructured finite element methods for both fluid and solid models. Nonlinear incremental iterative procedures are used to handle fluid-structure interactions. The governing finite element equations for both the solid and fluid models are solved by Newton-Raphson iteration method. Proper mesh was chosen to fit the shape of each component, the vessel, and the fluid domain. Finer mesh was used for thin plaque cap and components with sharp angles to get better resolution and handle high stress concentration behaviors. The artery was stretched axially and pressurized gradually to specified conditions. Mesh analysis was 
performed until differences between solutions from two consecutive meshes were negligible (less than $1 \%$ in $\mathrm{L}_{2}$-norm). Details of the computational models and solution methods can be found from Bathe [1,2] and Tang et al [6,7].

\section{Results and Conclusion}

Simulations were performed using several plaque samples under various conditions to investigate stress/strain behaviors and their correlations with pressure conditions, plaque morphology, plaque structure, and plaque vulnerability as determined by histopathological analysis. Only selected results are presented here.

(a) Slice 4

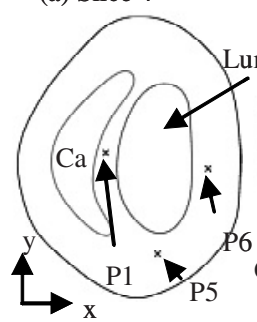

(b) Slice 12

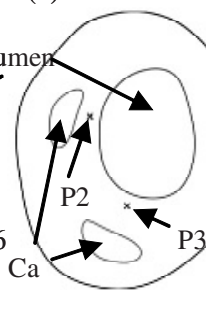

(c) Slice 20

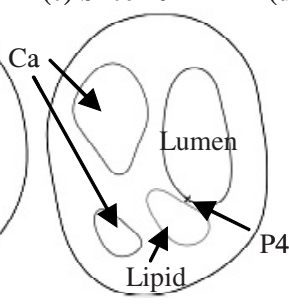

(d) Imposed Pressure Condition

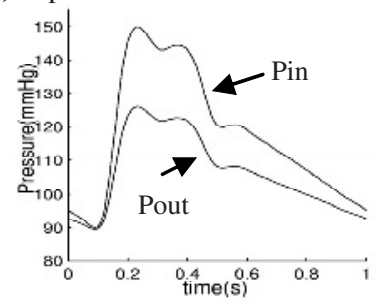

Fig. 2. Normal and critical points are selected to track stress/strain variations. P1: from calcification cap; P2: from a thicker Ca cap; P3: from a thicker Ca cap; P4: from a thin lipid cap (most vulnerable site); P5: normal point to observe stress-xx; P6: normal point to observe stress-yy. (a)-(c) give locations of the 6 points and 3 slices from the plaque sample (Fig. 1); (d) Imposed upstream pressure (Pin) and down-stream pressure (Pout) used for the simulation

(a) Stress-xx

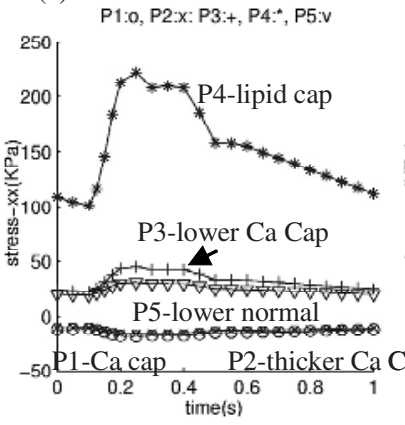

(b) Stress-yy

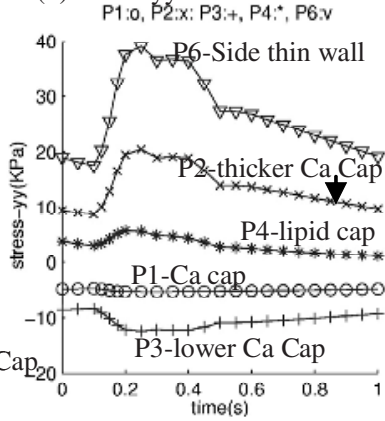

(c) Stress-xy

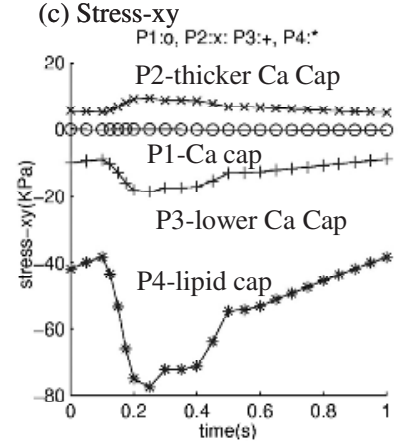

Fig. 3. Tracking of stress components at selected sites under pulsating pressure showing critical point from the thin lipid cap has much greater stress variations. (a) Normal stress in $\mathrm{x}$-direction (Stress-xx); (b) Normal stress in y-direction (Stress-yy); (c) Shear component (stress-xy) 


\subsection{Unsteady Stress/Strain Behaviors at Critical Sites Under Pulsating Pressure}

Blood flow is pulsatile. Blood vessel and atherosclerotic plaque are subjected to strong pulsating pressure conditions and the corresponding stress/strain behaviors are worthy investigating. We hypothesize that relative stress/strain variations in the plaque under pulsating pressure may correlate with plaque rupture risk. Using the plaque sample given by Fig. 1, several critical points were selected from various sites to observe stress/strain variations under pulsating pressure (Fig. 2). A human carotid pressure profile was scaled to $90-150 \mathrm{mmHg}$ range and used in the simulation. Down-stream pressure (Pout) was chosen so that flow rate was physiological (between $2-15 \mathrm{ml} / \mathrm{s}$ ). Fig. 3 shows that the thin cap point (Point 4) shows much greater $(>400 \%)$ stress variation than other points. Stress-yy from Point 4 was smaller because of the orientation of the cap. The large stress-yy variation from Point 6 (a normal point) is a false alarm caused by vessel thickness. These initial results indicate that stress/strain variations do carry useful information. However, they must be examined carefully, and plaque structure, cap thickness, component size and shape must be taken into consideration. Relevance of these findings with respect to plaque vulnerability needs to be established using histopathological and clinical data.

\subsection{Effect of Material Properties of Arteries and Plaque Components}

Using our models, we can change material parameters to perform sensitivity analysis to observe their impact on stress/strain distributions. Since calcification is much stiffer (almost rigid) than normal tissue, lipid is much softer than normal tissue, when their volumes are relatively small compared to the total plaque volume (i.e., they are not the main structure for the whole solid model), their stiffness changes (such as $50 \%$ reduction, $100 \%$ increase) do not alter stress/strain distributions much (less than $2 \%$ from some of our samples [7]); For vessel material test, when vessel stiffness was increased by $100 \%$ (by changing $\mathrm{c}_{1}$ and $\mathrm{D}_{1}$ values, using the plaque with small lipid pools), maximum stress values were almost unchanged (changes were less than $2 \%$ ) while maximum strain values were reduced by about $50 \%$. However, for a plaque sample with $30 \%$ calcification (shown by Fig. 1), contrary to what was observed from the plaque sample with smaller lipid pools, Fig. 4 and Table 1 show clearly that vessel material properties have considerable effect on maximum values of stress components (50\%-100\% differences). While calcification did not show much dominance in Stress-P1 and Stress-Xx plots, it is dominant in Stress-zz plot due to the 5\% axial stretch. Softer vessel case shows greater maximum stress values (about 40-50\% increase for most of the components, 65\% for Stress-xy, 92\% for Stress-yy) because calcification component took more load compared to vessel part. For the same reason, stiffer vessel case shows lower maximum stress values because calcification component was taking less load. It should be noted that these results are still of indicative nature because material properties for the vessel and plaque components were not measured directly for the samples simulated. Direct measurement of plaque component properties will be very desirable and will improve accuracy and reliability of computational findings. However, it is beyond the scope of our current research. 
(a) Slice 20

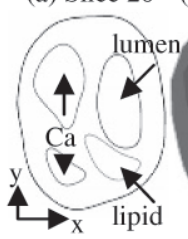

(b) S-p1,Base

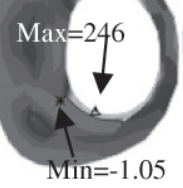

(c) S-xx.Base

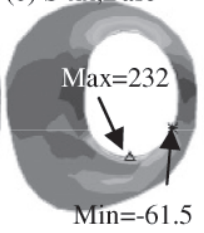

(d) S-zz,Base

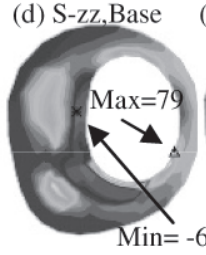

(e) S-zz Soft

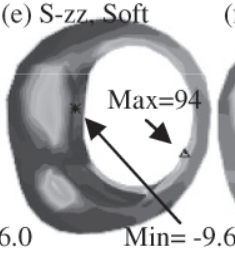

(f) S-zz, Stiff

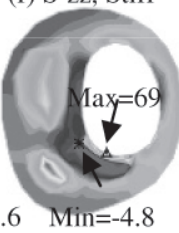

Fig. 4. Plots of selected stress components from the 3D model with different vessel material parameters showing that vessel material properties have considerable effect on stress distributions. Pin $=150 \mathrm{mmHg}$, Pout $=126 \mathrm{mmHg}$, pre-stretch=5\%. Material parameters were chosen so that the softer vessel is about $50 \%$ softer than baseline vessel and stiffer vessel is about $100 \%$ stiffer than baseline vessel. a) Slice 20 showing component shapes and positions; b) Stress-p1 from baseline model showing maximum stress at the thin plaque cap; c) Stress-xx from baseline model; d) Stress-zz from baseline model showing stress concentration in calcified region; e) Stress-zz from soft vessel model; f) Stress-zz from the stiff vessel model

Table 1. Maximum stress values from Slice 20 and Slice 4 of the large calcification plaque sample. Slice 4 is used because it does not contain lipid pool

\begin{tabular}{|l|l|l|c|c|c|c|c|}
\hline Cases & $\begin{array}{l}\text { S- } \\
\text { P1 }\end{array}$ & S-xx & S-xy & S-xz & S-yy & S-yz & S-zz \\
\hline Case 1: Baseline, 5\% stretch & 245 & 231 & 161 & 3.3 & 150 & 11.2 & 79.3 \\
\hline Case 2: Softer vessel & 373 & 355 & 266 & 4.9 & 288 & 16.0 & 94 \\
\hline Case 3: Stiffer vessel & 187 & 172 & 85 & 3.6 & 75 & 9.4 & 69.4 \\
\hline Case 4: Baseline,10\% Stretch & 247 & 229 & 161 & 4.4 & 146 & 13.9 & 107 \\
\hline Case 5: S4, baseline, no lipid & 259 & 241 & 82 & 6.0 & 51 & 6.4 & 51 \\
\hline
\end{tabular}

\subsection{Identifying Critical Stress/Strain Indicators for Plaque Vulnerability Assessment}

Atherosclerotic plaques have complex structures. Stress/strain tensors have 12 components (6 each), their distributions in plaque region have complex patterns, and their dynamic behaviors are even more difficult to analyze. We examine every stress/strain component in the plaque, under steady and pulsating pressures, seeking the right critical indicators. Some "candidate indicators" are: i) Global and local $\mathrm{max} / \mathrm{min}$ values of each stress/strain component corresponding to maximum pressure condition. These include local max/min values at thin plaque caps, sharp angle of lipid pools and other possible locations; ii) maximum stress/strain variations under pulsating pressure; iii) maximum stress/strain space variations (concentration) as represented by the derivatives of stress/strain tensors; iv) maximum cyclic stretch and compression as indicated by strain components under pulsating pressure.

The following scheme was used to identify possible critical stress/strain risk indicators. Histopathological analysis was performed to quantify plaque vulnerability (V) and 5 grades were assigned to each plaque: very stable, $\mathrm{V}=0$; stable, $\mathrm{V}=1$; slightly unstable, $\mathrm{V}=2$; unstable, $\mathrm{V}=3$; and vulnerable, $\mathrm{V}=4$, according to cap thickness, pool 
size, lumen surface conditions, and various cell counts (Fig. 5). Statistical analysis was performed to find correlation between "critical stress/strain indicators" and plaque vulnerability. Initial results have been obtained from 11 plaques using $2 \mathrm{D}$ models [8]. Our results indicate that there are positive correlations between Stress-P1 and plaque vulnerability, lipid pool area ratio, and a negative correlation with plaque cap thickness. More 3D samples are needed to perform the analysis using 3D results.

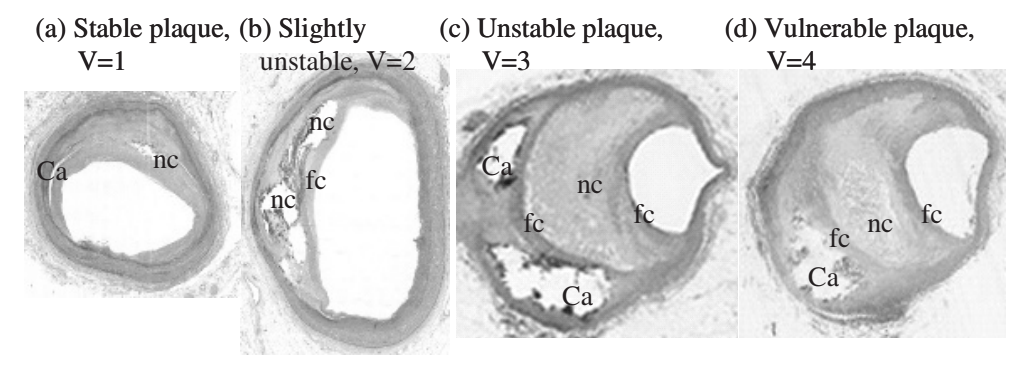

Fig. 5. Selected histological images of coronary plaques. V value is pathological classification of plaque vulnerability. $\mathrm{nc}=$ necrotic lipid core; $\mathrm{fc}=$ fibrous cap; $\mathrm{m}=$ matrix $; \mathrm{Ca}=$ calcification

\subsection{Conclusion}

Considerably higher stress/strain variations under pulsating pressure are observed at thin plaque cap. Calcification volume and vessel material properties have large impact on stress distributions. With sufficient validations, critical stress/strain indicators may be used for plaque vulnerability assessment.

Acknowledgement. This research was supported in part by NSF grant DMS-0072873 and a grant from the Rockefeller Foundation. $\mathrm{J}$ Zheng is supported in part by a Charles E. Culpeper Biomedical Pilot initiative grant 01-273.

\section{References}

1. Bathe, K.J.: Finite Element Procedures. Prentice Hall, New Jersey, (1996)

2. Bathe, K.J.: Theory and Modeling Guide, Vol I: ADINA; Vol II: ADINA-F, ADINA R\&D, Inc., Watertown, MA, (2002)

3. Huang, H., Virmani, R., Younis, H., Burke, A.P., Kamm, R.D., Lee, R.T.: The impact of calcification on the biomechanical stability of atherosclerotic plaques. Circulation (2001) 103: 1051-1056.

4. Naghavi M, et al. (57 co-authors including C. Yuan): From vulnerable plaque to vulnerable patient: a call for new definitions and risk assessment strategies: Part I. Circulation. (2003) 108(14):1664-72.

5. Schroeder W, Martin K, Lorensen B, The Visualization Toolkit, An Object-Oriented Approach To 3D Graphics, Prentice Hall, 2nd Edition, (1998)

6. Tang, D., Yang, C., Kobayashi, S., Ku, D.N.: Effect of a lipid pool on stress/strain distributions in stenotic arteries: 3D FSI models. J. Biomech. Engng, (2004) In press. 
7. Tang, D., Yang, C., Zheng, J., Woodard, P.K., Sicard, G.A., Saffitz, J.E., Yuan, C.: 3D MRI-Based Multi-Component FSI Models for Atherosclerotic Plaques a 3-D FSI model, Annals of Biomedical Engineering. (2004) 32(7):947-960.

8. Williamson SD, Lam Y, Younis HF, Huang H, Patel S, Kaazempur-Mofrad MR, Kamm RD. On the sensitivity of wall stresses in diseased arteries to variable material properties, J. Biomechanical Engineering, (2003) 125, 147-155.

9. Yuan C, Mitsumori LM, Beach KW, Maravilla KR. Special review: Carotid atherosclerotic plaque: noninvasive MR characterization and identification of vulnerable lesions. Radiology. (2001) 221:285-99. 